\title{
Second Order Large Deviation Estimates for Ferromagnetic Systems in the Phase Coexistence Region`
}

\section{Roberto H. Schonmann}

Mathematical Sciences Institute, Cornell University, Ithaca, NY14853 USA

Instituto de Mathemática e Estatística, Universidade de São Paulo, Caixa Postal 20570, 01000 São

Paulo, SP, Brasil

\begin{abstract}
We consider the $d$-dimensional Ising model with ferromagnetic nearest neighbor interaction at inverse temperature $\beta$. Let $M_{\Lambda}=|\Lambda|^{-1} \sum_{i \in \Lambda} \sigma_{i}$ be the magnetization inside a $d$-dimensional hyper cube $\Lambda, \mu_{+}$be the + Gibbs state and $m^{*}(\beta)$ be the spontaneous magnetization. For $\beta$ such that $m^{*}(\beta)>0$ we find a sufficient condition (easily verified to hold for large $\beta$ ) for $\mu_{+}\left(\left\{M_{\Lambda} \in[a, b]\right\}\right)$ to decay exponentially with $|\Lambda|^{(d-1) / d}$ when $-m^{*}<b<m^{*},-1 \leqq a<b$. For $d=2$ this sufficient condition is the exponential decay of a connectivity function. We also prove a partial converse to this result, obtain a sharper result for the magnetization on $d-1$ dimensional cross sections of the model and prove a similar result for $d=2,-m^{*}<a<b<m^{*}$, and $\beta$ large, when free boundary conditions are chosen outside $\Lambda$.
\end{abstract}

\section{Introduction}

We consider the Ising model with nearest neighbor interaction on a $d$-dimensional lattice $Z^{d}$. The spin at each point $x \in Z^{d}$ takes the value $\sigma_{x}= \pm 1$, and the formal energy of a spin configuration $\sigma$ is

$$
E(\sigma)=-(1 / 2) \sum_{x, y} J_{x, y} \sigma_{x} \sigma_{y}
$$

where $J_{x, y}=1$ if $x$ and $y$ are nearest neighbors and $J_{x, y}=0$ otherwise. A Gibbs measure at inverse temperature $\beta$ is any measure $\mu$ on $\{-1,1\}^{Z^{d}}$ such that for any choice of $a_{y}= \pm 1, y \in Z^{d}$, and any $x \in Z^{d}$

$$
\begin{aligned}
& \mu\left(\left\{\sigma: \sigma_{x}=a_{x}\right\} \mid\left\{\sigma: \sigma_{y}=a_{y} \text { for } y \neq x\right\}\right) \\
& \quad=\exp \left((\beta / 2) \sum_{y} J_{x, y} a_{x} a_{y}\right) /\left(\exp \left((\beta / 2) \sum_{y} J_{x, y} a_{y}\right)+\exp \left(-(\beta / 2) \sum_{y} J_{x, y} a_{y}\right)\right),
\end{aligned}
$$

$\mu$ almost surely.

* Work partly supported by the U.S. Army Research Office 
Let $\mu_{+}$(respectively $\mu_{-}$) be the Gibbs measure obtained as a limit of Gibbs measures on finite regions with + (respectively - ) boundary conditions. Set

$$
m^{*}=m^{*}(\beta)=\int \sigma_{0} d \mu_{+}
$$

and

$$
\beta_{c}=\inf \left\{\beta \geqq 0: m^{*}(\beta)>0\right\}=\inf \left\{\beta \geqq 0: \mu_{-} \neq \mu_{+}\right\} .
$$

As is well known, $0<\beta_{c}<\infty$ for $d \geqq 2$.

In this paper we are interested in the asymptotics of the $\mu_{+}$distribution of the spin per site in large regions. For any finite $\Lambda \subset Z^{d}$, let $|\Lambda|$ be its cardinality and define the random variable

$$
M_{\Lambda}=M_{\Lambda}(\sigma)=|\Lambda|^{-1} \sum_{x \in \Lambda} \sigma_{x}
$$

Set also

$$
p_{\Lambda}(a, b)=\mu_{+}\left\{\sigma: M_{\Lambda}(\sigma) \in[a, b]\right\}
$$

Consider now a sequence $\left(\Lambda_{n}\right)=(\Lambda)$, where $\Lambda_{n}$ is a cube of sides $n$ and therefore $\left|\Lambda_{n}\right|$ $\rightarrow \infty$ as $n \rightarrow \infty$. For convenience of notation we will choose $\Lambda_{n}=\left\{x \in Z^{d}: 1 \leqq x_{i} \leqq n\right.$, $i=1, \ldots, d\}$. Since $\mu_{+}$is ergodic with respect to translations, $M_{\Lambda} \rightarrow m^{*} \mu_{+}$a.s. In particular $p_{\Lambda}(a, b) \rightarrow 0$ if $m^{*} \notin[a, b]$. Furthermore the following large deviation property is by now well known $[2,4,5,9,14]$.

$$
|\Lambda|^{-1} \log \mu_{+}\left\{\sigma: M_{\Lambda}(\sigma) \in[a, b]\right\} \rightarrow-\inf _{a \leqq m \leqq b} \varphi(m)
$$

for any $-1 \leqq a<b \leqq 1$, where

$$
\varphi(m)=\sup _{h \in \mathbb{R}}\{h m-p(h)+p(0)\}
$$

and

$$
p(h)=\lim _{\Lambda}|\Lambda|^{-1} \log \sum_{\sigma \in\{-1,1\}^{\Lambda}} \exp \left(\beta\left(\sum_{x, y \in \Lambda} J_{x, y} \sigma_{x} \sigma_{y}+h \sum_{x \in \Lambda} \sigma_{x}\right)\right)
$$

is $-\beta$ times the thermodynamic pressure when an external field $h$ is applied.

$p(h)$ and $\varphi(m)$ are convex. For $\beta>\beta_{c}, \varphi(m)$ is zero on $\left[-m^{*}, m^{*}\right]$ and positive outside this interval. Therefore $p_{\Lambda}(a, b)$ goes to zero exponentially fast with $|\Lambda|$ if and only if $a<b<-m^{*}$ or $m^{*}<a<b$. The same large deviation estimates, with the same function $\varphi(\cdot)$, hold for any other Gibbs measure and also if one considers Gibbs measures in $\Lambda$ with some boundary conditions outside $[9,14]$. Therefore it is desirable to obtain further information about $p_{\Lambda}(a, b)$, mainly for $-m^{*}<a<b$ $<m^{*}$, when surface effects should be important and the results should depend on the Gibbs measure considered.

We find a sufficient condition (easily verified to hold for large $\beta$ ) for $p_{\Lambda}(a, b)$ to decay exponentially fast with the surface of $\Lambda$ when $-m^{*}<b<m^{*},-1 \leqq a<b$, i.e., if $\partial \Lambda$ is the set of points in $\Lambda$ which are neighbors to points in the complement of $\Lambda$, then there exist constants $A_{1}, A_{2}, c_{1}, c_{2}>0$ such that

$$
A_{1} e^{-c_{1}|\partial \Lambda|} \leqq p_{\Lambda}(a, b) \leqq A_{2} e^{-c_{2}|\partial \Lambda|} .
$$


The lower bound in (1.1) is easy to prove by a well known argument which can be applied to any finite range ferromagnetic system. Basically the argument is that a circuit of - spins has only a cost which is exponential in its cardinality and is enough to change the state in the region which it surrounds roughly to $\mu_{-}$. For completeness we state this result and prove it in the next section.

Proposition 1. For any $\beta$ such that $m^{*}>0$ and any $-m^{*}<a<b<m^{*}$, there exist $A$, $c>0$ such that $p_{\Lambda}(a, b) \geqq A e^{-c|\partial \Lambda|}$.

In order to define the sufficient condition mentioned above we will think of the configurations as specified by the contours on the dual lattice $(Z+1 / 2)^{d}$ which separate + and - spins (see for instance $[7,12]$ and references there). Provided that there is no infinite cluster of - spins the configuration is completely specified by the contours if we assume that spins that are not surrounded by any contour are + . Contours not surrounded by any other contour are called outer contours. We will call a contour positive (negative) if the outside spins adjacent to it are positive (negative). In the absence of infinite clusters of - spins the outer contours are positive $\mu_{+}$a.s.

We will also need the following definitions. A chain is a finite sequence $\left(x_{1}, \ldots, x_{2}\right)$ of distinct points in $Z^{d}$ such that $x_{i-1}$ and $x_{i}$ are neighbors for $i=2, \ldots, n$. A subset $R \subset Z^{d}$ is connected if, for all pairs $x, y$ of points in $R$, there is a chain formed by points in $R$ having $x, y$ as terminal points.

Let $\Omega$ be the set of subsets $S$ of $Z^{d}$ such that $x_{d}=0$ for all $x \in S$. For $S \in \Omega$ consider the event.

$$
C_{S}=\{S \text { is surrounded by a contour }\} .
$$

The sufficient condition that we have is the absence of infinite clusters of - spins and the existence of $A, c>0$ such that

$$
\mu_{+}\left(C_{S}\right) \leqq A e^{-c\|S\|}, \quad \text { for any } S \in \Omega,
$$

where

$$
\|S\|=\inf \left\{|R|: R \subset Z^{d}, R \text { is connected, } S \subset R\right\} .
$$

In dimension two this condition is equivalent to the requirement that with respect to $\mu_{+}$the probability that the points $(0,0)$ and $(n, 0)$ are surrounded by a same contour decays exponentially with $n$.

For each dimension $d \geqq 2$ there exists a finite $\beta_{d}^{\prime}$ (for instance $\beta_{2}^{\prime}=\log 3$ ) such that if $\beta>\beta_{d}^{\prime}$, then a Peierls type of estimate can be used (for details see [7] or [12]) to show that the probability of the occurrence of a contour of cardinality $l$ surrounding a fixed point decreases exponentially with $l$. It follows that for $\beta>\beta_{d}^{\prime}(1.2)$ holds. (See also Remark 2 at the end of this introduction.)

Our main result is

Theorem 1. If (1.2) holds, then (1.1) holds for any $-m^{*}<b<m^{*},-1 \leqq a<b$.

We will prove also a partial converse to Theorem 1 . Let

$$
T_{n}=\left\{x \in Z^{d}: 0 \leqq x_{i} \leqq n, i=1, \ldots, d-1, x_{d}=0\right\} \in \Omega .
$$

Then it is easy to prove that 


\section{Proposition 2.}

$$
\lim _{n} n^{-d+1} \log \mu_{+}\left(C_{T_{n}}\right)=\sup _{n} n^{-d+1} \log \mu_{+}\left(C_{T_{n}}\right):=-\Delta_{\beta, d}=-\Delta .
$$

It is clear that if (1.2) holds then $\Delta>0$. We will prove that

Theorem 2. If $\Delta=0$ then for any $-1 \leqq a<-m^{*},-m^{*}<b<m^{*}$ and any $\varepsilon>0$, $p_{\Lambda}(a, b) \geqq e^{-\varepsilon|\delta \Lambda|}$ for $\Lambda$ large enough.

This is only a partial converse to Theorem 1 because $a$ and $b$ are more restricted $\left(-m^{*}\right.$ must belong to $\left.(a, b)\right)$ and because (1.2) must fail for the particular regions $T_{n}$. In $d=2$ this second restriction is not important since in this case (1.2) and $\Delta>0$ are clearly equivalent. (But see Remark 2 below.)

Before proving Theorem 2 in Sect. 3 we will obtain many properties of $\Delta$ and an alternative definition of this quantity for $d=2$ which relates it in a closer form to the correlation length defined in percolation theory $[1,13]$.

Theorems 1 and 2 relate percolation problems for $\mu_{+}$(see Sect. 3) to other properties of this measure. For more on this approach the reader is referred to $[3,8,15]$.

On the way to proving Theorem 1 we will prove a result which is interesting on its own right. Let $\left\{\eta_{x}, x \in Z^{d-1}\right\}$ be defined by

$$
\eta_{x}=\sigma_{\left(x_{1}, x_{2}, \ldots, x_{d-1}, 0\right)} \text {. }
$$

i.e., $\eta_{x}$ are the spins on a $d-1$ dimensional "cross section" of $Z^{d}$. Remember the definition of $T_{n}$ and set

$$
X_{n}=X_{n}(\sigma)=M_{T_{n}}(\sigma)=n^{-d+1} \sum_{\substack{1 \leqq x_{1} \leqq n \\ i=1, \ldots, d-1}} \eta_{x} .
$$

Lemma 1. If (1.2) holds and $m<m^{*}$, then there exist $A, c>0$ such that

$$
\mu_{+}\left\{\sigma: X_{n}(\sigma) \leqq m\right\} \leqq A \exp \left(-c n^{d-1}\right) .
$$

Once Lemma 1 is proven it is very easy to finish the proof of Theorem 1 (see the end of Sect. 2).

Combining Lemma 1 with results in [10] leads to a stronger result for the random field $\left\{\eta_{i}: i \in Z^{d-1}\right\}$, which is proven in Sect. 4 :

Theorem 3. If (1.2) holds, then there exists a convex function $\theta:[-1,1] \rightarrow[0, \infty)$ such that $\theta(x)=0$ if and only if $x=m^{*}$ and

for any $-1 \leqq a<b \leqq 1$.

$$
n^{-d+1} \log \mu_{+}\left\{\sigma: X_{n}(\sigma) \in[a, b]\right\} \rightarrow-\inf _{a \leqq x \leqq b} \theta(x)
$$

This behavior contrasts with the large deviations for $M_{\Lambda}$, where the function $\varphi(\cdot)$ is 0 on $\left[-m^{*}, m^{*}\right]$.

In Sect. 5 we consider Gibbs measures on finite regions with boundary conditions. Let $\mu_{\Lambda^{+}}$and $\mu_{\Lambda 0}$ be respectively the Gibbs measures on $\{-1,1\}^{\Lambda}$ when the boundary condition is + or free [7]. Define

$$
p_{\Lambda+}(a, b)=\mu_{\Lambda+}\left\{\sigma: M_{\Lambda}(\sigma) \in[a, b]\right\},
$$

and analogously for $p_{\Lambda 0}(\cdot, \cdot)$. 
It is easy to see that Theorem 1 holds with $p_{\Lambda_{+}}(\cdot, \cdot)$ in the place of $p_{\Lambda}(\cdot, \cdot)$ (the lower bound can be prove in the same way and the upper bound follows from that for $p_{\Lambda}(\cdot, \cdot)$ by $\left.\mathrm{FKG}\right)$.

In two dimensions at large $\beta$ we obtain a similar result for free boundary conditions:

Theorem 4. For $d=2, \beta>\log 3$ and any $-m^{*}<a<b<m^{*}$ there exist positive constants $A_{1}, A_{2}, c_{1}, c_{2}$ such that

$$
A_{1} e^{-c_{1}|\partial \Lambda|} \leqq p_{\Lambda 0}(a, b) \leqq A_{2} e^{-c_{2}|\partial \Lambda|} .
$$

The analogue of (1.4) is clearly not true for all boundary conditions. For instance if the spins outside $\Lambda$ are positive on the upper half plane and negative otherwise, then $M_{\Lambda}$ concentrates around $1 / 2$.

Inequality (1.4) appeared as a conjecture, for a more general class of systems, in [4]. For $d \geqq 3$ it is not clear for us whether (1.4) holds even when (1.1) does. The approach that we will use to prove Theorem 4 fails for $d \geqq 3$, but can be used to prove a weaker statement in this case. We discuss this point at the end of Sect. 4.

Remark 1. Two basic questions that we leave open are

a) Is (1.1) true in any dimension for any $\beta>\beta_{c}$ ? For $d \geqq 3$ the present approach is not likely to work with this generality because for $\beta$ slightly greater then $\beta_{c},(1.2)$ probably fails. In fact one expects that in this regime there is percolation of - spins in the + phase $\mu_{+}$.

b) Can (1.1) be sharpened by proving that in fact $|\partial \Lambda|^{-1} \log p_{\Lambda}(a, b)$ converges for every $a$ and $b$ ? If this is the case, is the limit related to other quantities of physical interest like surface tension?

Remark 2. After this paper was finished, J. T. Chayes, L. Chayes and the author proved that (1.2) holds for $d=2$ for any $\beta>\beta_{c}$; therefore (1.1) follows in this case with this generality. Moreover in $d=2(1.4)$ holds for any $\beta>\beta_{c}$. These results will be the subject of a future publication.

Remark 3. When this paper was finished, we learned also that J. Bricmont and S. Olla proved (1.1) for any $d \geqq 2$ for large enough $\beta$. They obtained their results independently of ours and at about the same time, using a completely different technique. Comparing the two approaches we can say that ours has the advantage that it permitted us to prove (1.1) in $d=2$ up to $\beta_{c}$ (see Remark 2 above). But on the other hand our method does not give the sharper result mentioned in part $b$ of Remark 1, while there is hope that their approach can be improved to give this result.

\section{Main Result}

Warning. Throughout this paper $A$ and $c$ will represent positive constants, but their values may change from expression to expression.

Proof of Proposition 1. Let $\delta$ be such that

$$
-\delta m^{*}+(1-\delta) m^{*}=(a+b) / 2
$$


and $l_{n}$ be the integer part of $n \delta$. Remember that

$$
\Lambda=\Lambda_{n}=\left\{x \in Z^{d}: 1 \leqq x_{i} \leqq n, i=1, \ldots, d\right\},
$$

and define

$$
\Lambda_{n}^{-}=\left\{x \in \Lambda_{n}: x_{1} \leqq l_{n}\right\}, \quad \Lambda_{n}^{+}=\left\{x \in \Lambda_{n}: x_{1}>l_{n}\right\} .
$$

Consider the event

$$
E_{n}=\left\{\sigma: \sigma_{x}=-1 \quad \forall x \in \partial \Lambda_{n}^{-} \text {and } \sigma_{y}=+1 \quad \forall y \in \partial \Lambda_{n}^{+}\right\} .
$$

Then for any Gibbs measure $\mu$,

where

$$
\mu\left(E_{n}\right) \geqq \alpha^{\left|\partial \Lambda_{n}^{+}\right|+\left|\partial \Lambda_{n}^{-}\right|} \geqq \alpha^{2\left|\partial \Lambda_{n}\right|},
$$

But

$$
\alpha=e^{-2 \beta} /\left(e^{2 \beta}+e^{-2 \beta}\right)>0 .
$$

$$
p_{\Lambda}(a, b) \geqq \mu_{+}\left(E_{n}\right) \cdot \mu_{+}\left(\left\{\sigma: M_{\Lambda}(\sigma) \in[a, b]\right\} \mid E_{n}\right) .
$$

Therefore we will be done once we prove that the second factor above goes to 1 as $|\Lambda| \rightarrow \infty$.

Set $\Gamma=\Lambda_{n}^{-} \backslash \partial \Lambda_{n}^{-}$. By the Markov property of $\mu_{+}$, conditioning on $E_{n}$, the spins inside $\Gamma$ only "see" the-boundary condition at $\partial \Lambda_{n}^{-}$. Therefore for any $\varepsilon>0$,

$$
\begin{aligned}
& \mu_{+}\left(\left\{\sigma: M_{\Gamma}(\sigma) \in\left(-m^{*}-\varepsilon,-m^{*}+\varepsilon\right)\right\} \mid E_{n}\right) \\
& \quad=\mu_{\Gamma^{-}}\left(\left\{\sigma: M_{\Gamma}(\sigma) \in\left(-m^{*}-\varepsilon,-m^{*}+\varepsilon\right)\right\} \mid E_{n}\right) .
\end{aligned}
$$

By FKG

$$
\mu_{\Gamma^{-}}\left(\left\{\sigma: M_{\Gamma}(\sigma) \geqq-m^{*}+\varepsilon\right\}\right) \leqq \mu_{-}\left(\left\{\sigma: M_{\Gamma}(\sigma) \geqq-m^{*}+\varepsilon\right\}\right) .
$$

But the right hand side of this inequality vanishes as $n \rightarrow \infty$ by the ergodicity of $\mu_{-}$. It is also known (by the methods in [9], or Theorem 5.2 in [14]) that

$$
(\partial \Gamma)^{-1} \log \mu_{\Gamma^{-}}\left(\left\{\sigma: M_{\Gamma}(\sigma) \leqq-m^{*}-\varepsilon\right\}\right) \rightarrow-\varphi\left(-m^{*}-\varepsilon\right),
$$

as $n \rightarrow \infty$. Since the free energy $\varphi(m)$ is strictly positive for $m<m^{*}$, it follows that

$$
\mu_{\Gamma^{-}}\left(\left\{\sigma: M_{\Gamma^{\prime}}(\sigma) \leqq-m^{*}-\varepsilon\right\}\right) \rightarrow 0 .
$$

Putting the facts above together we have

$$
\mu_{+}\left(\left\{\sigma: M_{\Gamma}(\sigma) \in\left(-m^{*}-\varepsilon,-m^{*}+\varepsilon\right)\right\} \mid E_{n}\right) \rightarrow 1 .
$$

Analogously, setting $\bar{\Gamma}=\Lambda_{n}^{+} \backslash \partial \Lambda_{n}^{+}$, one can prove

$$
\mu_{+}\left(\left\{\sigma: M_{\bar{\Gamma}}(\sigma) \in\left(m^{*}-\varepsilon, m^{*}+\varepsilon\right)\right\} \mid E_{n}\right) \rightarrow 1 .
$$

To finish the proof it is enough to choose $\varepsilon=(b-a) / 4$.

Observe that the proof above applies to any Gibbs measure, even when boundary conditions outside $\Lambda$ are fixed.

One of the basic ingredients in the proof of Lemma 1 is a mixing property of the random field $\left(\eta_{i}: i \in Z^{d-1}\right)$. This property will be stated and proved in Lemma 2 below. Theorem 3 in [12] states an even stronger mixing condition, but there is a mistake in its proof (the next to last phrase on p. 81 of that paper contains a wrong statement). 
Moreover the mixing condition stated there cannot be true (see for instance Proposition 2 of [15]). Nevertheless we used many of Martin Löf's ideas in that paper to prove our Lemma 2.

Next we will prove Lemma 1 in the case $d=2$. The case of larger dimension does not present greater conceptual difficulties, but the notation and details would obscure the argumentation. We will make some remarks about the modifications in the proof for $d>2$ after proving Lemma 2 .

Proof of Lemma 1. Set $\varepsilon=m^{*}-m$. By the ergodicity of $\mu_{+}$it is possible to choose an $L \in \mathbb{N}$ such that

$$
\mu_{+}\left\{\sigma: X_{L}(\sigma) \leqq m^{*}-\varepsilon / 3\right\} \leqq 3^{-3 / \varepsilon} / 2 .
$$

For $N \in\{1,2, \ldots\}$ to be chosen later, define the regions

$$
\begin{aligned}
& R_{i}=\left\{x \in Z^{2}: 1 \leqq x_{1}-(i-1) N L \leqq L, x_{2}=0\right\}, \\
& S_{j}=\bigcup_{i=1}^{j} R_{i} .
\end{aligned}
$$

Consider the random variables

$$
\begin{aligned}
\zeta_{i} & = \begin{cases}1 & \text { if } M_{R_{i}}(\sigma)>m^{*}-\varepsilon / 3, \\
0 & \text { otherwise }\end{cases} \\
Y_{j} & =j^{-1} \sum_{i=1}^{j} \zeta_{i} .
\end{aligned}
$$

By Lemma 2 below and our choice of $L$ it is possible to choose $N$ large enough so that for any $k$ and $i_{1}<i_{2}<\cdots<i_{k}$,

$$
\mu_{+}\left(\zeta_{i_{k}}=0 \mid \zeta_{i_{1}}=\cdots=\zeta_{i_{k-1}}=0\right) \leqq 3^{-3 / \varepsilon}
$$

Then

$$
\mu_{+}\left(\zeta_{i_{1}}=\cdots=\zeta_{i_{k}}=0\right) \leqq\left(3^{-3 / \varepsilon}\right)^{k} .
$$

On the other hand, by translation invariance, for $n=N L j, j \in\{1,2, \ldots\}$,

$$
\begin{aligned}
\mu_{+}\left\{\sigma: X_{n}(\sigma)\right. & \left.\leqq m^{*}-\varepsilon\right\} \leqq N \mu_{+}\left\{\sigma: M_{S_{j}}(\sigma) \leqq m^{*}-\varepsilon\right\} \\
& \leqq N \mu_{+}\left\{\sigma: Y_{j} \leqq 1-\varepsilon / 3\right\} .
\end{aligned}
$$

Where we used the fact that on $\left\{\sigma: Y_{j}>1-\varepsilon / 3\right\}$,

$$
M_{S_{j}}(\sigma) \geqq(1-\varepsilon / 3)\left(m^{*}-\varepsilon / 3\right)-\varepsilon / 3>m^{*}-\varepsilon .
$$

But $Y_{j} \leqq 1-\varepsilon / 3$ implies that for at least $[j \varepsilon / 3]$ different values of $i \in\{1, \ldots, j\}, \zeta_{i}=0$. Since there are not more than $2^{j}$ ways of choosing $[j \varepsilon / 3]$ out of $j$, it follows from $(2.1)$ that

$$
\mu_{+}\left\{\sigma: Y_{j} \leqq 1-\varepsilon / 3\right\} \leqq 2^{j} \cdot\left(3^{-3 / \varepsilon}\right)^{[j \varepsilon / 3]} \leqq 3^{3 / \varepsilon} \cdot(2 / 3)^{j}=A e^{-c n}
$$

for some positive $A$ and $c$. Together with (2.2) this finishes the proof when $n$ is a multiple of $N L$. The extension for all $n$ is routine.

Remember that an event $E$ is called positive (respectively negative) if its indicator 
function is a coordinatewise increasing (respectively decreasing) function of the configurations.

Lemma 2. Let $R_{i}$ and $S_{j}$ be as in the proof of Lemma 1. Assume that (1.2) holds. Then, for fixed $L$, given $\varepsilon>0$ it is possible to find $N$ such that

$$
\left|\mu_{+}(F \mid E)-\mu_{+}(F)\right| \leqq \varepsilon,
$$

for any pair of negative events $E$ and $F$ which depend respectively on $\left\{\sigma_{x}: x \in S_{j}\right\}$ and $\left\{\sigma_{x}: x \in R_{j+1}\right\}$ for some $j$.

Proof. Set

$$
\bar{E}=\left\{\sigma: \sigma_{x}=-1 \forall x \in S_{j}\right\}
$$

By the FKG inequality,

$$
\mu_{+}(F) \leqq \mu_{+}(F \mid E) \leqq \mu_{+}(F \mid \bar{E}) .
$$

Let $G_{j}(x)$ be the event that every $R_{i}, i=1, \ldots, j$, is surrounded by a contour and the innermost such collection of contours also surrounds the point $x \in R_{j+1}$. Decompose $G_{j}(x)$ into the events $(k=1, \ldots, j)$

$$
\begin{aligned}
G_{j, k}(x)= & \left\{G_{j}(x) \text { occurs and the contour that surrounds } x\right. \\
& \text { surrounds also } R_{k} \text { but does not surround any } R_{i} \text { with } \\
& 1 \leqq i<k\} .
\end{aligned}
$$

Write also

Now

$$
H_{r, s}=\left\{\sigma: \sigma_{x}=-1 \forall x \in R_{i}, r \leqq i \leqq s\right\}
$$

$$
\mu_{+}\left(G_{j, k}(x) \mid \bar{E}\right)=\frac{\mu_{+}\left(G_{j, k}(x) \cap H_{1, k-1} \cap H_{k, j}\right)}{\mu_{+}\left(H_{k, j} \cap H_{1, k-1}\right)} .
$$

By the FKG inequality

$$
\begin{aligned}
\mu_{+}\left(H_{k, j} \cap H_{1, k-1}\right) & \geqq \mu_{+}\left(H_{k, j}\right) \mu_{+}\left(H_{1, k-1}\right) \\
& \geqq \alpha^{L(j-k+1)} \mu_{+}\left(H_{1, k-1}\right)
\end{aligned}
$$

where $\alpha$ was defined in the proof of Proposition 1. On the other hand, if $\gamma$ is a dual circuit which surrounds $R_{k}$ and $x$ and does not surround $R_{i}$ for $1 \leqq i<k$,

$\mu_{+}\left(\left\{\gamma\right.\right.$ is the innermost contour which surrounds $\left.\left.R_{k}\right\} \cap H_{k, j} \cap H_{1, k-1}\right)$

$\leqq \mu_{+}\left(\{\gamma\right.$ is a positive contour $\left.\} \cap H_{1, k-1}\right)$

$=\mu_{+}(\{\gamma$ is a positive contour $\}) \cdot \mu_{+}\left(H_{1, k-1} \mid\{\gamma\right.$ is a positive contour $\left.\}\right)$

$\leqq \mu_{+}(\{\gamma$ is a positive contour $\}) \cdot \mu_{+}\left(H_{1, k-1}\right)$.

In the last inequality we used the fact that $\gamma$ does not surround $R_{i}, i=1, \ldots, k-1$; therefore by the Markov property of $\mu_{+}$and by FKG this inequality follows. Summing over $\gamma$ gives

$$
\begin{aligned}
& \mu_{+}\left(G_{j, k}(x) \cap H_{1, k-1} \cap H_{k, j}\right) \\
& \quad \leqq \mu_{+}\left(\left\{\text {There is a contour which surrounds } R_{k} \text { and } x\right\}\right) \cdot \mu_{+}\left(H_{1, k-1}\right) .
\end{aligned}
$$


From (2.4), (2.5), (2.6), and (1.2),

Hence

$$
\begin{aligned}
\mu_{+}\left(G_{j, k}(x) \mid \bar{E}\right) & \leqq A \exp [-c(j-k+1) N L] \cdot \alpha^{-L(j-k+1)} \\
& =A \exp [(-c N-\log \alpha) L(j-k+1)] .
\end{aligned}
$$

$$
\mu_{+}\left(G_{j}(x) \mid \bar{E}\right) \leqq \sum_{k=1}^{j} \mu_{+}\left(G_{j, k}(x) \mid \bar{E}\right) \leqq \sum_{r=1}^{\infty} A \exp [(-c N-\log \alpha) L r] .
$$

From these inequalities it is clear that there exists $N_{0}$ such that if $N>N_{0}$ then

Set

$$
\mu_{+}\left(G_{j}(x) \mid \bar{E}\right) \leqq \varepsilon / L
$$

$$
G_{j}=\bigcup_{x \in R_{j+1}} G_{j}(x)
$$

Then by (2.7), for $N>N_{0}$

Now

$$
\mu_{+}\left(G_{j} \mid \bar{E}\right) \leqq \varepsilon
$$

$$
\begin{aligned}
\mu_{+}(F \mid \bar{E})= & \mu_{+}\left(F \mid \bar{E} \cap G_{j}\right) \cdot \mu_{+}\left(G_{j} \mid \bar{E}\right) \\
& +\mu_{+}\left(F \mid \bar{E} \cap\left(G_{j}\right)^{c}\right) \cdot \mu_{+}\left(\left(G_{j}\right)^{c} \mid \bar{E}\right) .
\end{aligned}
$$

But on $\bar{E} \cap\left(G_{j}\right)^{c}, R_{j+1}$ is isolated from $S_{j}$ by positive contours $\mu_{+}$a.s. Therefore by the Markov and FKG properties

So, by (2.8) and (2.9)

$$
\mu_{+}\left(F \mid \vec{E} \cap\left(G_{j}\right)^{c}\right) \leqq \mu_{+}(F)
$$

$$
\mu_{+}(F \mid \bar{E}) \leqq \mu_{+}(F)+\varepsilon,
$$

and using (2.3) the proof is complete.

In $d>2$ one can prove Lemma 1 in an analogous way using the following definitions of $R_{i}$ and $S_{j}, i, j \in N^{d-1}$ :

$$
\begin{aligned}
& R_{i}=\left\{x \in Z^{d}: 1 \leqq x_{r}-\left(i_{r}-1\right) N L \leqq L, r=1, \ldots, d-1 ; x_{d}=0\right\}, \\
& S_{j}=\bigcup_{\substack{1 \leqq i_{r} \leqq j \\
r=1, \ldots, d-1}} R_{i} .
\end{aligned}
$$

Lemma 2 can then be stated with $E$ depending on $\left\{\sigma_{x}: x \in S_{j} \backslash R_{i}\right\}$ for some $j$ and $i$ and $F$ depending on $\left\{\sigma_{x}: x \in R_{i}\right\}$. Note that when one proves the analogue of (2.7) then, there is an extra polynomial (and therefore harmless) factor in $r$, due to the number of sets $R_{k}$ which are at a distance $r$ from $R_{i}$.

Proof of Theorem 1. By Lemma 1 and translation invariance

$$
\begin{aligned}
p_{\Lambda}(a, b) & \leqq \mu_{+}\left\{\sigma: M_{\Lambda}(\sigma) \leqq b\right\} \\
& \leqq \mu_{+}\left(\bigcup_{x_{d}=1}^{n}\left\{\sigma: n^{-1} \sum_{\substack{1 \leqq x_{\imath} \leqq n \\
l=1, . ., d-1}} \sigma_{\left(x_{1}, \ldots, x_{d}\right)} \leqq b\right\}\right) \\
& \leqq n \mu_{+}\left\{\sigma: X_{n}(\sigma) \leqq b\right\} \leqq A e^{-c|\partial \Lambda|},
\end{aligned}
$$

if $b<m^{*}$. Combining this with Proposition 1 completes the proof. 


\section{Partial Converse}

Proof of Proposition 2. This proof is an example of a well known argument $[1,13]$; for $d=2$ it goes as follows. Define the event

$$
C(n, m)=\left\{\left\{x \in Z^{2}: n \leqq x_{1} \leqq m, x_{2}=0\right\} \text { is surrounded by a contour }\right\} .
$$

Now realize that $C(n, m)$ are negative events $[1,6]$ since the substitution of some spins + by - spins will not permit us to remove all the contours that surround $C(n, m)$. Since $\mu_{+}$satisfies the FKG inequalities and is invariant under translations

$$
\begin{aligned}
\mu_{+}(C(0, n+m)) & =\mu_{+}(C(0, n) \cap C(n, n+m)) \geqq \mu_{+}(C(0, n)) \cdot \mu_{+}(C(n, n+m)) \\
& =\mu_{+}(C(0, n)) \cdot \mu_{+}(C(0, m)),
\end{aligned}
$$

from which Proposition 2 follows by superadditivity.

The proof is similar in higher dimensions (see Lemma A2.4 in [9]) or the proof of part (a) of Theorem 1 in [10]).

Before proving Theorem 2 we need more properties of $\Delta$. First some definitions. We will say that a set $S \subset Z^{d}$ surrounds a set $R \subset Z^{d}$ if there is only a finite number (possible zero) of sites $x \in Z^{d}$ such that there is a chain which does not intercept $S$ and has $x$ and a point in $R$ as terminal.

Consider the events

$$
\begin{aligned}
D_{n}^{(h)}= & \left\{\text { There is } S \subset\left\{x \in Z^{d}:\left|x_{d}\right| \leqq h\right\} \text { which surrounds } T_{n}\right. \\
& \text { and such that } \left.\sigma_{x}=-1 \text { for every } x \in R\right\} .
\end{aligned}
$$

Then, for fixed $n, D_{n}^{(h)}$ is an increasing sequence which converges to $D_{n}^{(\infty)}=C_{T_{n}}$. Another basic property of $D_{n}^{(h)}$ is that for each $h$ and $n$ they are negative events.

\section{Proposition 3.}

a) $\lim _{n} n^{-d-1} \log \mu_{+}\left(D_{n}^{(h)}\right)=\sup _{n} n^{-d+1} \log \mu_{+}\left(D_{n}^{(h)}\right):=-\Delta_{h}$,

b) $\lim _{h \rightarrow \infty} \Delta_{h}=\Delta$.

Proof. The proof of (a) is analogous to that of Proposition 2. The proof of (b) is the same argument used to prove a similar statement about self avoiding random walks in [1]. It is clear that $\Delta_{h}$ decreases with $h$ and $\Delta_{h} \geqq \Delta$. On the other hand by Proposition 1 and part (a) above, for any $\varepsilon>0$ there exists an $n$ such that

$$
\exp \left(-n^{d-1}(\Delta+\varepsilon)\right) \leqq \mu_{+}\left(C_{T_{n}}\right)=\lim _{h \rightarrow \infty} \mu_{+}\left(D_{n}^{(h)}\right) \leqq \lim _{h \rightarrow \infty} \exp \left(-n^{d-1} \Delta_{h}\right) .
$$

Therefore $\Delta+\varepsilon \geqq \lim _{h \rightarrow \infty} \Delta_{h}$.

One can prove (see [12] and the proof of Lemma 2) that if $\Delta>0, \mu_{+}$has exponentially decaying correlations. The quantity $\Delta^{-1}$ is a kind of "correlation surface" (correlation length in $d=2[1,13]$ ). In $d=2, \Delta$ can be defined by the same type of events used to define correlation length for independent subcritical percolation $[1,13]$. For this purpose say that two sites are $\left(^{*}\right)$ neighbors if they are neighbors or are such that both their coordinates differ by one unit. Define $\left(^{*}\right)$ chain 
as was done for chain. Set

$$
\begin{aligned}
\bar{D}_{n}= & \left\{\text { There is a } *^{*}\right) \text { chain } R \text { such that }(0,0) \text { and }(n, 0) \text { are } \\
& \text { their terminal points and } \left.\sigma_{n}=-1 \text { for every } x \in R\right\} .
\end{aligned}
$$

Then the relation

$$
\lim _{n} n^{-1} \log \mu_{+}\left(\bar{D}_{n}\right)=-\Delta,
$$

can be easily proven using Proposition 3 .

Proof of Theorem 2. Set

$$
\tilde{\Lambda}_{n}(h)=\left\{x \in Z^{d}: h \leqq x_{i} \leqq n-h, i=1, \ldots, d\right\} .
$$

As $\Delta=0$, it follows from Proposition 3 that given $\varepsilon>0$ it is possible to choose $h$ such that

$$
\mu_{+}\left(D_{n}^{(h)}\right)>\exp \left(-\varepsilon n^{d-1} / 4 d\right) .
$$

By FKG, translation invariance and right-angle rotation invariance of $\mu_{+}$, it follows that if

$E_{n}=\left\{\right.$ there is $R \subset Z^{d} \backslash \tilde{\Lambda}(h)$ which surrounds $\tilde{\Lambda}_{n}(h)$ and $\sigma_{x}=-1$ for every $\left.x \in R\right\}$, then

$$
\mu_{+}\left(E_{n}\right)>\exp \left(-\varepsilon n^{d-1} / 2\right) .
$$

Now the same argument used in Proposition 1, based on the Markov property of $\mu_{+}$, shows that

$$
p_{\Lambda}(a, b) \geqq(1 / 2) \exp \left(-\varepsilon n^{d-1} / 2\right)>\exp \left(-\varepsilon n^{d-1}\right)
$$

for $n$ large; finishing the proof.

\section{Cross Sections}

Proof of Theorem 3. Since the random field $\left(\eta_{i}: i \in Z^{d-1}\right)$ satisfies the FKG relations, we can apply Theorem 1 in [10], i.e., there exists a convex function $\theta:[-1,+1]$ $\rightarrow[0, \infty)$ such that $(1.3)$ is satisfied for any $-1 \leqq a<b \leqq 1$ such that

$$
\max \{\theta(a), \theta(b)\}>0 .
$$

Therefore we have to show that $\theta(m)=0$ only if $m=m^{*}$. This follows from Lemma 1 and the similar fact that for any $m>m^{*}$ there exist $A, c>0$ such that

$$
\mu_{+}\left\{\sigma: X_{n}(\sigma) \geqq m\right\} \leqq A \exp \left(-c n^{d-1}\right) .
$$

One way to prove this result is analogous to the proof of Lemma 1 (Lemma 2 with $E$ and $F$ positive follows from Theorem 1 in [12]. This theorem was stated there for $\beta>\beta_{d}^{\prime}$, but it is clear from its proof that it holds whenever (1.2) is satisfied.) Readers which are familiar with interacting particle systems [11] can see also that the methods used to prove Theorem 2 in [10] imply this result since there are stochastic Ising models (Glauber dynamics) corresponding to the interactions we are 
considering which are translation invariant attractive spin systems (see Chap. 4 of [11]).

\section{Free Boundary Conditions}

Proof of Theorem 4. The proof of Proposition 1 applies independently of boundary conditions and gives the lower bound. We prove now the upper bound.

For each $\Lambda_{n}$, let $\Gamma_{n}(\delta)$ be another square with the same center and with side $[(1-2 \delta) n]$. Choose $a^{\prime} \in\left(-m^{*}, a\right)$ and $b^{\prime} \in\left(b, m^{*}\right)$. Clearly it is possible to find a $\delta$ now such that for $n$ large enough

where

$$
p_{\Lambda 0}(a, b) \leqq \mu_{\Lambda_{n} 0}\left(Q_{n}\right)
$$

$$
\begin{aligned}
Q_{n} & =Q_{n}^{+} \cap Q_{n}^{-}, \\
Q_{n}^{+} & =\left\{\sigma: M_{\Gamma_{n}(\delta)}(\sigma) \geqq a^{\prime}\right), \\
Q_{n}^{-} & =\left\{\sigma: M_{\Gamma_{n}(\delta)}(\sigma) \leqq b^{\prime}\right) .
\end{aligned}
$$

For each choice of sign we define the events

$W_{n}^{ \pm}=\left\{\right.$There is a connected set $R$ contained in $\Lambda_{n} \backslash \Gamma_{n}(\delta)$ which intercepts $\partial \Lambda_{n}$ and surrounds $\Gamma_{n}(\delta)$ and $\sigma_{x}= \pm 1$ for all $\left.x \in R\right\}$

Clearly

$$
W_{n}=W_{n}^{+} \cup W_{n}^{-} \text {. }
$$

$$
\mu_{\Lambda_{n} 0}\left(Q_{n}\right) \leqq \mu_{\Lambda_{n} 0}\left(Q_{n} \mid W_{n}^{+}\right)+\mu_{\Lambda_{n} 0}\left(Q_{n} \mid W_{n}^{-}\right)+\mu_{\Lambda_{n} 0}\left(\left(W_{n}\right)^{c}\right)
$$

By FKG and the Markov property,

$$
\mu_{\Lambda_{n} 0}\left(Q_{n} \mid W_{n}^{+}\right) \leqq \mu_{\Lambda_{n} 0}\left(Q_{n}^{-} \mid W_{n}^{+}\right) \leqq \mu_{+}\left(Q_{n}^{-}\right) .
$$

Analogously

$$
\mu_{\Lambda_{n} 0}\left(Q_{n} \mid W_{n}^{-}\right) \leqq \mu_{-}\left(Q_{n}^{+}\right) .
$$

The result will now follow from (5.1), (5.2), (5.3) and Theorem 1, once we prove that for some positive $A, c$,

$$
\mu_{\Lambda_{n} 0}\left(\left(W_{n}\right)^{c}\right) \leqq A e^{-c n}
$$

This can be proven by the same arguments used in [12] to prove his Lemma 2. First realize that if $W_{n}$ does not occur, then there is a contour which starts at $\partial \Lambda_{n}$ (more precisely between two points of $\partial \Lambda_{n}$ ), reaches $\Gamma_{n}(\delta)$ and eventually returns to $\partial \Lambda_{n}$. By a Peierls type of estimate, the probability of each such contour $\gamma$ is bounded above by $2 e^{-\beta|\gamma|}$. (For details of this type of estimate see the proof of Lemma 1 in [22]. The factor 2 appears because with free boundary conditions there are two spin configurations for each configuration of contours.) Since the number of contours of length $l$ starting at a given point is not larger than $3^{l}$ and the number of starting points for the contour is $\left|\partial \Lambda_{n}\right|=4 n$, one gets

$$
\left.\mu_{\Lambda_{n} 0}\left(\left(W_{n}\right)^{c}\right) \leqq A n \exp (-\beta-\log 3) \delta n\right) .
$$

From which (5.4) follows. 
The approach used to prove Theorem 4 for $d=2$ does not work for $d \geqq 3$ for the following reason. With analogous definitions of $\Gamma_{n}(\delta)$ and $W_{n}$, it is clear that $\left(W_{n}\right)^{c}$ occurs if there are two chains, one of + spins and one of - spins, each one connecting $\partial \Lambda_{n}$ to $\partial \Gamma_{n}(\delta)$. Therefore

$$
\mu_{\Lambda_{n} 0}\left(\left(W_{n}\right)^{c}\right) \geqq \alpha^{2 n \delta},
$$

where $\alpha$ was defined in the proof of Proposition 1 . Hence, for every $\delta>0, \mu_{\Lambda_{n} 0}\left(\left(W_{n}\right)^{c}\right)$ does not decay exponentially with $\left|\partial \Lambda_{n}\right|$. Nevertheless, the same method used to prove (5.4) above can be used to prove that in any dimension $\mu_{\Lambda_{n} 0}\left(\left(W_{n}\right)^{c}\right)$ does decay exponentially with $n$, provided that $\beta>\beta_{d}^{\prime}$. It follows then, under this condition, that for $-m^{*}<a<b<m^{*}$ there are $A, c>0$ such that

$$
p_{\Lambda 0}(a, b) \leqq A e^{-c n} \text {. }
$$

Acknowledgements. We learned many of the ideas used in this paper while working with Rick Durrett on similar questions for percolation and the contact process. We would also like to thank M. Aizenman, J. Bricmont, H. Kesten, J. Lebowitz, and S. Olla for many discussions on large deviations and related subjects. This paper benefited from the criticism of a referee. We are thankful to A. Gandolfi for pointing out a mistake in a previous version of this paper.

\section{References}

1. Chayes, J. T., Chayes, L.: Percolation and random media. To appear in Les Houches Session XLIII 1984: Critical phenomena, random systems and gauge theories. Osterwalder, K., Stora, R. (eds.). Amsterdam: Elsevier

2. Comets, F.: Grandes deviations pour des champs de Gibbs sur $Z^{d}$. CR. Acad. Sci. 303, Ser. I, 511-513 (1986)

3. Coniglio, A, Nappi, C., Peruggi, F., Russo, L.: Percolation and phase transitions in the Ising model. Commun. Math. Phys. 51, 315-323 (1976); Percolation points and critical point in the Ising model. J. Phys. A: Math. Gen. 10, 205-218 (1977)

4. Ellis, R.: Large deviations for the spin per site in ferromagnetic models. Preprint, University of Massachusetts 1986

5. Fölmer, H., Orey, S.: Large deviations for the empirical field of a Gibbs measure. Preprint 1986

6. Fortuin, C. M., Kasteleyn, P. W., Ginibre, J.: Correlation inequalities on some partially ordered sets. Commun. Math. Phys. 22, 89-103 (1971)

7. Gallavotti, G.: Instabilities and phase transitions in the Ising model, a review. Riv. Nuovo Cimento 2 , 133-169 (1972)

8. Higuchi, Y.: A weak version of RSW Theorem for the two-dimensional Ising model. Contemp. Math. Vol. 41, 207-214, A.M.S., 1985, Durrett, R. (ed.)

9. Lanford, O. E.: Entropy and equilibrium states in classical statistical mechanics. Lecture Notes in Physics, Vol. 20, Berlin, Heidelberg, New York: Springer 1973, pp. 1-113

10. Lebowitz, J. L., Schonmann, R. H.: Pseudo free energies and large deviations for non-Gibbsian FKG measures. Preprint, Rutgers 1986

11. Liggett, T. M.: Interacting particle systems. Berlin, Heidelberg, New York: Springer 1985

12. Martin-Löf, A.: Mixing properties, differentiability of the free energy and central limit theorem for a pure phase in the Ising model at low temperature. Commun. Math. Phys. 32, 75-92 (1973)

13. Nguyen, B. G.: Correlation lengths for percolation processes. PhD. thesis (UCLA)

14. Olla, S.: Large deviations for Gibbs random fields. Preprint, Rutgers 1986

15. Russo, L.: The infinite cluster method in the two-dimensional Ising model. Commun. Math. Phys. 67, 251-266 (1979)

Communicated by M. Aizenman

Received November 24, 1986; in revised form March 10, 1987 
Notes added in proof

1) Recently M. Aizenman, J. Bricmont, and J. Lebowitz proved that in large dimensions - spins do percolate in the + phase for certain values of $\beta$.

2) The results mentioned in Remark 2 will appear in: J. T. Chayes, L. Chayes, R. H. Schonmann: Exponential Decay of Connectivities in the Two-Dimensional Ising Model. To appear in the Journal of Statistical Physics. 